\title{
Recent Advances on 5G Resource Allocation Problem using PD-NOMA
}

\author{
Sally Ismail ${ }^{1,3}$, Fabio D'Andreagiovanni ${ }^{1,2}$, Hicham Lakhlef ${ }^{1}$, Youcef Imine ${ }^{1}$ \\ ${ }^{1}$ Heudiasyc UMR 7253, Sorbonne Universités, Université de Technologie de Compiègne, CNRS, \\ CS 60319, 60203 Compiègne, France \\ ${ }^{2}$ National Centre for Scientific Research (CNRS), France \\ ${ }^{3}$ Faculté de Génie, Université Libanaise, Liban \\ Email: sally.ismail@etu.utc.fr, \{d.andreagiovanni, hicham.lakhlef, youcef.imine\}@hds.utc.fr
}

\begin{abstract}
G is the fifth generation technology standard for cellular networks, which is already being deployed and offering many advantages for communications, such as the high degree of flexibility, the possibility of management and control of systems and resources, thus allowing to host and execute services in one or more distinct network slices, etc. Non-orthogonal multiple access (NOMA) is being considered as a key enabling technique for $5 \mathrm{G}$ cellular systems. The earlier generations are becoming less practical with the emergent requirements such as very high spectral efficiency, very low latency, supporting diverse quality of services (QoS). Researchers are working on different techniques to fulfill these requirements, and NOMA is one of the essentials due to its capacity to increase spectrum efficiency. NOMA schemes exploit channel gain differences to serve multiple users concurrently, by superimposing multiple users' information signals at the transmitter side. In this paper, we review, analyze and classify recent papers on resource allocation problem in 5G networks, and then we discuss the proposed solutions. In particular, the papers are classified into two main categories: power/energy-efficient and rate-optimal. For each paper, the objective, optimization method and main results are discussed. Finally, some open challenges are highlighted.

Index Terms-Non-orthogonal multiple access (NOMA), 5G cellular, power allocation, resource allocation, optimization, energy-efficient, power-efficient, rate-optimal.
\end{abstract}

\section{INTRODUCTION}

In existing cellular network systems, orthogonal multiple access (OMA) techniques have been used: frequency division multiple access (FDMA) in first generation (1G), time division multiple access (TDMA) in 2G, code division multiple access (CDMA) in 3G and Orthogonal frequency division multiple access (OFDMA) in 4G. The common between these multiplexing techniques is the orthogonality feature, which means that different users don't interfere when they are accessing the network resources. As for wireless networks such as the 5G and beyond 5G (B5G) are expected to support extremely high data rates and a very large number of users, which is not achievable using OMA. To toggle this problem, non-orthogonal multiple access (NOMA) schemes have been introduced, where multiple users are allowed to share the same resource block (time/frequency) and are alternatively separated in other domains, with a cost of additional complexity at the receivers. NOMA offers several following features: improved spectral efficiency, improved user fairness, low transmission latency, higher cell-edge throughput [1], [2].

Different categories of NOMA has been studied, the main two are power domain NOMA (PD-NOMA) and code domain NOMA (CD-NOMA) [3].

In power-domain multiplexing, different users are allocated different power coefficients according to their channel conditions. In code-domain multiplexing, different users are allocated different codes and multiplexed over the same timefrequency resources, such as multi-user shared access (MUSA) [4], sparse code multiple access (SCMA) [5], and low-density spreading (LDS) [6]. On the other hand, resource allocation problem is widely studied in cellular networks. Given the available resources (time, frequency,...), the way you choose to assign these resources to the users will heavily affect the performance of your system. Resource allocation is a key area to achieve the full potential of communication systems with NOMA [7]. With the objective of achieving a certain goal, the resource allocation can vary between: power allocation, channel assignment, transmission scheduling, user selection and joint schemes of these techniques [8].

In the current work, we will be focusing on recent papers handling the resource allocation problem for PD-NOMA.

The rest of the paper is organized as follows: in section II, we provide a brief description of NOMA. Section III surveys the papers on resource allocation. In section IV, a comparative table is provided in addition to some open challenges. Finally, section $\mathrm{V}$ concludes the paper.

\section{BACKGROUND}

PD-NOMA was first introduced to improve the spectral efficiency of wireless networks by allowing multiple users to share both time and frequency domains, while allocating different power levels to each user at the transmitter side, whereas all the receivers (i.e. users) receive their desired signals along with the interference caused by the messages of other users. To obtain the desired signal, each successive interference cancellation (SIC) receiver first decodes all dominant interference (i.e. the ones that are stronger then the receiver's desired signal) and then subtracts them from the superposed signal [9]. To clarify more the idea, let's take the case of two-user down-link (DL) NOMA, i.e. the case where a base 
station (BS) is sending signals to two users, as shown in figure 1 .

In typical PD-NOMA scenario, at the transmitter side, both signals of the two users are superposed upon each other with different power allocation. Usually, the signal of the user with strong channel gain will be allocated with the lower power, and the one of the user with weak channel gain will be allocated with the higher power. Then, at the strong user's receiver, the signal of the weak user, having power higher than his own, will have a high signal-to-noise ratio (SNR), meaning that the strong user can successfully decode and subtract the signal of the weak user before decoding his own signal (i.e.performing SIC). For the weak user side, at his receiver, the signal of the strong user, having less power than his own, will be considered as noise when decoding his signal, so he will be decoding his signal without SIC.

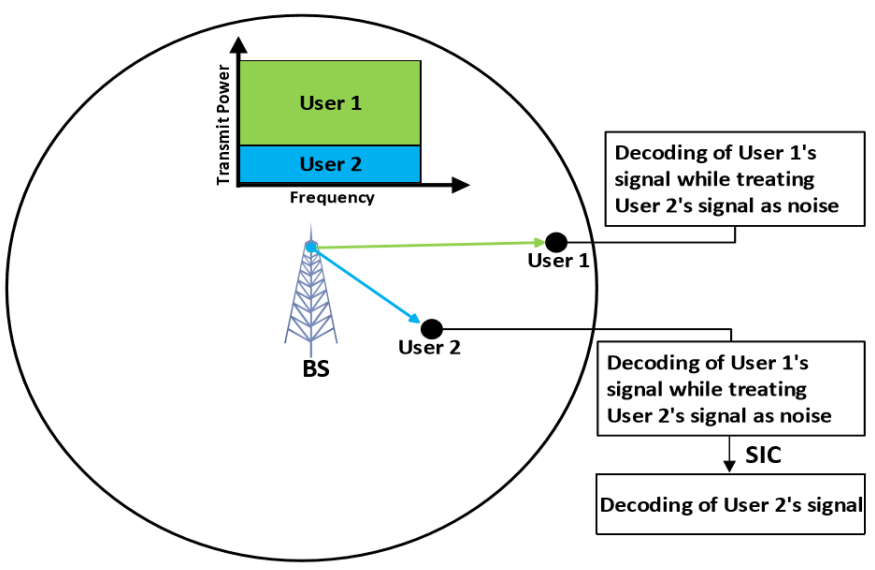

Figure 1. An illustration of a two-user down-link PD-NOMA scheme [4]

For the two-user up-link (UL) scenario, where the two users are sending their signal to the $\mathrm{BS}$, the signals are also sent simultaneously with different transmit power. The power share of each user is again based on their channel conditions with respect to the BS. Then, after the transmission, the BS receives the superposed signal containing both users' signal, and starts by decoding the strongest signal, subtracting it from the superposed signal and then decodes the other signal.

\section{RESOURCE ALLOCATION}

Resource allocation is a key area to achieve the full potential of communication systems with NOMA.

Based on what's explained earlier about PD-NOMA, we can see that power allocation and grouping or clustering of users are two important, but not the only, steps for NOMA schemes. It is also worth mentioning that the optimal user clustering usually requires an exhaustive search and may not always be affordable, and for this reason, researchers resort to low complexity solutions to solve the problem through heuristic algorithms which may lead to unpredictable results, or alternatively they use [2]:
- The monotonic optimization approach: the monotonic nature of the objective function and constraints allows researchers to limit the exhaustive search to a much smaller region of the feasible set; this accordingly simplifies the problem and can lead to the optimal solution but still with relatively high complexity.

- The combinatorial relaxation approach: the selection and association of a particular user to a particular cluster is realized by a binary variable; by relaxing this variable to a continuous one, the original non-convex problem can be transformed to a convex one and solved optimally through classic Lagrangian dual method, but this relaxation results in a performance gap between the original problem and the relaxed one.

- The matching theory approach: which is often utilized to deal with problems that have beneficial relationship parties.

- The game theory approaches: which transform the clustering problem into a game.

The choice of which resource allocation to use depends on the type of communication system that will be applied. Therefore, it is necessary to choose the optimal strategy according to the specific scenario [8]. In addition, the performance analysis metrics changes depending on the application, it could be the bit error rate (BER), outage probability, etc...

In the current work, the resource allocation will be classified into two main categories:

- Power-efficient or Energy-efficient resource allocation whose goal is to find an optimal scenario in which the energy generated for communication is managed sustainability. Hence, the effect on the environment, economy, living beings, etc is minimized [10].

- Rate-optimal resource allocation whose goal is to optimize the achievable rate and thus the spectral efficiency.

\section{A. Power/Energy-efficient resource allocation}

While some papers addressed the power-efficient NOMA and others the energy-efficient NOMA problems, both categories reside under the goal of achieving an environment friendly technology.

1) Power-efficient resource allocation:

The authors in [11] address the power-efficient resource allocation problem in virtualized wireless networks (VWNs).

Virtualization of a wireless network is a promising technique, in which the physical wireless infrastructure is shared among multiple service providers, also called slices. Aiming to increase the spectrum and infrastructure efficiency, the main issue in a VWN is to prevent the harmful effects of users of one slice to the users of other slices. The authors wanted to see if NOMA could help improving the network performance of a VWN in terms of power efficiency. So, they compared two transmission modes: NOMA where the whole frequency band of interest is shared among users, and OFDMA where the specific bandwidth is divided into a set of sub-carriers, and each sub-carrier is allocated to a maximum of one user at a time. The objective was to minimize the total transmit 
power in a VWN while maintaining the minimum required capacity for each slice. The formulated problem, for both cases, was non-convex which is challenging to solve. For NOMA, an efficient algorithm was proposed to solve the problem, where complementary geometric programming and variable relaxation were used to convert the problem into the geometric programming. As for OFDMA, relaxation technique was applied and then Lagrange dual function was used to solve the problem.

The simulation results demonstrated that NOMA is more power-efficient than OFDMA in various scenarios, and specifically, the power efficiency was improved by up to $45-54 \%$ with NOMA as compared to OFDMA.

In [8], the authors proposed power-efficient resource allocation for multi-carrier (MC) NOMA systems with quality of service (QoS) constraints under statistical channel state information at the transmitter (CSIT).

In conventional multi-carrier (MC) systems, a given radio frequency band is divided into multiple sub-carriers (i.e. multicarrier) and each sub-carrier is allocated to at most one user. For NOMA systems, each sub-carrier could be assigned to more than one user.

Usually when dealing with resource allocation problems, perfect CSIT is assumed, and this is the case for a vast range of papers on resource allocation, but in practice, perfect CSIT is hard to obtain. So the authors considered an imperfect CSIT scenario, and due to this fact, a SIC policy taking consideration of QoS requirements is proposed, which only allows one user to perform SIC. And based on the considered policy, only two users per sub-carrier were allowed, and the resource allocation was formulated for MC-NOMA systems to minimize the total transmit power.

The formulated problem is a mixed combinatorial non-convex problem, and a sub-optimal solution is proposed to solve the power allocation and the user scheduling problems separately.

- For the power allocation problem: the user scheduling policy is supposed given, and the power allocation is performed on each sub-carrier independently. The selection of optimal power allocation in incorporates the SIC decoding policy implicitly to achieve minimum power consumption.

- For the user scheduling problem: an algorithm was proposed based on the agglomerative hierarchical clustering.

As for the results, the proposed user scheduling method provides a significant power saving compared to the random scheduling, and achieves a performance close to the full search scheduling. It achieves a close-to-optimal performance and significantly outperforms a conventional orthogonal multiple access (OMA) scheme.

2) Energy-efficient resource allocation:

Energy-efficient NOMA will be a key ingredient in the upcoming $5 \mathrm{G}$ mobile network design to cater for the goal of achieving a low cost, self-sustaining, environment-friendly wireless network. Mathematically, energy efficiency is defined as the amount of information that can be reliably transmitted per Joule of consumed energy; which is denoted using units of bits-per-Joule [10].

In [12], the authors proposed an energy-efficient user scheduling or pairing and power allocation for a DL of NOMA wireless network.

The problem was formulated with an objective of maximizing the total energy efficiency of the system while respecting the total power constraint. The authors started by finding the optimal user scheduling, and then, based on that, they searched for the optimal power allocation.

For the channel pairing, the authors considered the case of two users per sub-channel. They chose to use the channel state sorting-pairing algorithm, which pairs the best channel condition user with the worst channel condition user, with the goal of increasing the system sum rate and decreasing the interference between users of the same sub-channel.

After the user pairing is achieved, the BS needs to perform two stages power allocation: power allocation for different subchannels, and power allocation for different users in each subchannel. For the first stage, the author considered having a subchannel with a fixed power allocation and he finds the optimal power distribution between the two users that maximizes the sub-channel transmit rate. As for the second stage, and due to the complexity of the problem (non-convex problem), they transformed it into Dinkelbach representation and proposed an algorithm to find the optimal solution. Finally, simulations of the proposed solution were compared to OFDM scheme and it was proven that the proposed NOMA solution is more energyefficient than OFDM.

In [13], the authors did a comparison between NOMA and TDMA to find out which scheme is more spectral and energy efficient for wireless powered IoT networks. In these kind of networks, multiple energy-limited devices first harvest energy in the DL and then transmit information in the UL (see figure 2).

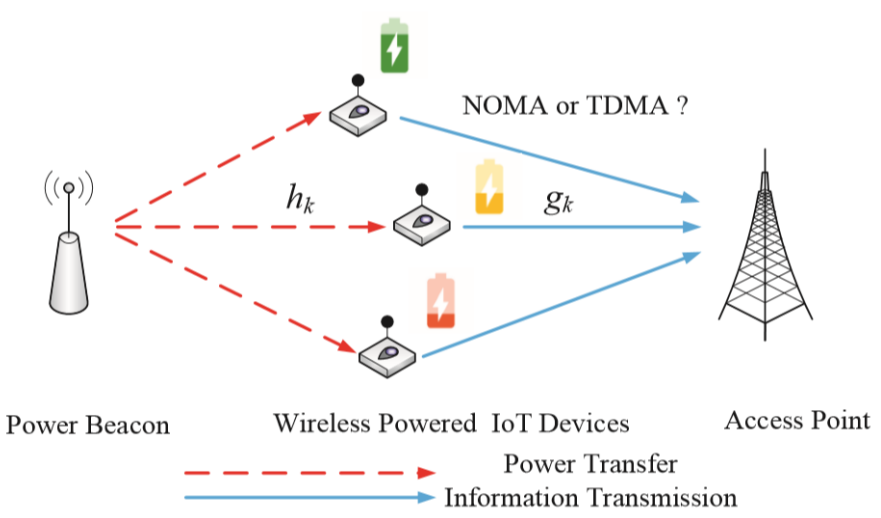

Figure 2. System model of a wireless powered IoT network [13]

They started by deriving the optimal DL time and power allocation for the spectral efficiency maximization in both cases, and then calculating the corresponding energy consumption and spectral efficiency at these optimal values. For TDMA, 
the formulated problem was a convex optimization problem satisfying Slater's condition, so the optimal solution was found using Lagrange dual method. The NOMA formulation also had the same structure, so the optimal solution was the same with difference in the constant parts. And then, for these optimal values, energy consumption and spectral efficiency were calculated. In conclusion, it was found that NOMA needs more (or equal) DL time than TDMA, while consuming more (or equal) energy and achieving less spectral efficiency.

In [14], the authors proposed using NOMA in backsacatter communication (BackCom) for green IoT networks. BackCom is a key enabler technique for wireless power communication networks (WPCN), where power is transmitted to IoT devices through radio frequency (RF) signals. A BackCom system is composed of backscatter nodes (BNs) and a reader that transmits a single-tone sinusoidal RF signal. Upon receiving the reader $\mathrm{RF}$ signal, the $\mathrm{BN}$ reflects it back to the reader by impedance mismatching at the antenna input side. In a BackCom system, the reader may receive reflected signals from multiple BNs during the UL data transmission, which can lead to interference at the receiver. So, to tackle this problem, the authors employed a hybrid TDMA scheme with PD-NOMA for UL communication.

A sensor field of $\mathrm{K}$ IoT sensors (BNs) and a reader are considered. The reader transmits power to the sensors located in the reader annular coverage zone, and than these sensors can backscatter the incident wave to transmit data back to the reader. After receiving all the backscattered signals from the BNs, the reader transmits the decoded data to a cellular BS. To implement the proposed PD-NOMA, the reader splits the annular coverage zone into $\mathrm{N}$ number of virtual subregions as shown in fig.4, and sensors from different subregions are multiplexed together in a single mini-timeslot of $\mathrm{T}$ seconds. It should be mentioned that since the BNs are passive devices, and cannot actively alter the transmitted power as needed in conventional PD-NOMA systems, reflection coefficiens are used instead such as to give the best system performance.

To test the proposed scheme, it is compared to an OMAbased TDMA scheme where each sensor is allocated a single dedicated mini-timeslot for transmission. The performance is rated in terms of system outage probability and throughput. The results showed that the proposed PD-NOMA scheme outperforms the conventional TDMA scheme and increases the system throughput.

\section{B. Rate-optimal resource allocation}

In [15], the authors studied the resource allocation and user scheduling problem for a DL NOMA network. They intended to jointly optimize the sub-channel assignment and power allocation to maximize the weighted total sum-rate while taking into account user fairness. User fairness is considered in terms of capacity, so a weight factor is introduced for each user to adjust its priority when allocating resources.

The formulated problem was proven to be NP-hard, so, in order to solve it, they proposed to decouple the power allocation and sub-channel sub-problems and proposed an iterative algorithm in which the power and sub-channel allocations are performed in an iterative way to obtain a joint solution of the problem:

- To solve the power allocation problem, they supposed that the sub-channel allocation is settled, so the problem could be formulated as geometric programming and solved using interior point methods.

- For the sub-channel allocation sub-problem, it was formulated as many-to-many matching game with externalities, and to solve it, two matching algorithms were introduced.

With the above stated, the solution of the problem is the following:

It starts with an initialization phase were the BS allocates equal power to each user over each sub-channel and the weight factor for each user is set as inversely proportional to the average rate of user in previous time slots. In the resource allocation phase, sub-channel assignment and power allocation are iteratively performed as to obtain a joint solution.

The simulations' results proved that the proposed algorithm outperformed traditional OFDMA scheme in terms of both total sum-rate and user fairness.

In [16], the authors investigated the resource allocation for MC-NOMA systems.

In this paper, the authors aim to treat the MC-NOMA systems, so they tried and managed to find a resource allocation (optimal joint power and sub-carrier allocation policy) algorithm for the weighted system throughput problem of MC-NOMA systems. The formulated problem was a mixed combinatorial non-convex problem, and it was optimally solved using monotonic optimization based resource allocation algorithm. The authors also proposed a low-complexity sub-optimal algorithm that has a polynomial time complexity, to balance between complexity and system performance. Finally, the simulation results proved that the proposed algorithm outperforms conventional MC-OMA in terms of system performance, also, the sub-optimal algorithm achieves similar performance as the optimal one.

In [17], the authors treated the problem of weighted sumrate (WSR) maximization in a DL MC-NOMA with cellular power constraint. This problem is important due to the fact that it offers trade-offs between sum-rate performance and user fairness.

For these problems, two types of power constraints are considered in the literature: cellular power constraint and individual power constraint. In this paper the authors only considered the cellular power constraint, which represent the total available power at the BS.

This is a joint sub-carrier and power allocation optimization problem, and to solve it, the authors divided the problem into two polynomial time solvable sub-problems to take advantage of each one's particular structure, and find an efficient solution, and to finally solve the initial problem by combining the two solutions. The two sub-problems are:

- The single-carrier user selection (SCUS): only one subcarrier is considered with its power budget, and the 
Table I

COMPARATIVE TABLE OF THE ANALYZED PAPERS

\begin{tabular}{|c|c|c|c|c|c|}
\hline Reference & Classification & Link Type & Resources & Optimization Method & Main Finding(s) \\
\hline$[11]$ & Power-efficient & DL & $\begin{array}{l}\text { joint power and sub-carrier al- } \\
\text { location }\end{array}$ & $\begin{array}{l}\text { Iterative algorithm based on } \\
\text { CGP }\end{array}$ & $\begin{array}{l}\text { The proposed algorithm is more } \\
\text { power-efficient than OFDMA }\end{array}$ \\
\hline$[8]$ & Power-efficient & DL & $\begin{array}{l}\text { Power allocation and user } \\
\text { scheduling }\end{array}$ & $\begin{array}{l}\text { Sub-optimal solution that treats } \\
\text { the two problems separately }\end{array}$ & $\begin{array}{l}\text { Close-to-optimal performance } \\
\text { and the solution outperforms } \\
\text { a conventional orthogonal } \\
\text { multiple access (OMA) scheme }\end{array}$ \\
\hline [12] & $\begin{array}{l}\text { Energy- } \\
\text { efficient }\end{array}$ & DL & $\begin{array}{l}\text { Joint power allocation and user } \\
\text { scheduling }\end{array}$ & $\begin{array}{l}\text { Channel state sorting-pairing al- } \\
\text { gorithm and algorithm based on } \\
\text { Dinkelbach for power allocation }\end{array}$ & $\begin{array}{l}\text { The proposed solution is more } \\
\text { energy-efficient than OFDM }\end{array}$ \\
\hline [13] & $\begin{array}{l}\text { Spectral } \\
\text { and Energy- } \\
\text { efficient }\end{array}$ & UL and DL & Joint time and power allocation & $\begin{array}{l}\text { Solution based on Lagrange } \\
\text { dual method }\end{array}$ & TDMA outperformed NOMA \\
\hline [14] & $\begin{array}{l}\text { Energy- } \\
\text { efficient }\end{array}$ & UL & Power allocation & N/A & $\begin{array}{l}\text { The proposed solution outper- } \\
\text { formed TDMA scheme and in- } \\
\text { creased the system throughput. }\end{array}$ \\
\hline$[15]$ & Rate-optimal & DL & $\begin{array}{l}\text { Joint power and sub-carrier al- } \\
\text { location and user scheduling }\end{array}$ & $\begin{array}{l}\text { Interior point method and joint } \\
\text { subchannel and power algo- } \\
\text { rithm }\end{array}$ & $\begin{array}{l}\text { The proposed solution outper- } \\
\text { formed OFDMA in terms of } \\
\text { sum-rate and user fairness }\end{array}$ \\
\hline [16] & Rate-optimal & DL & $\begin{array}{l}\text { Joint power and sub-carrier al- } \\
\text { location }\end{array}$ & $\begin{array}{l}\text { Algorithm based on monotonic } \\
\text { optimization and low complex- } \\
\text { ity sub-optimal algorithm }\end{array}$ & $\begin{array}{l}\text { The proposed algorithm outper- } \\
\text { forms MC-OMA, and the sub- } \\
\text { optimal algorithm is close to } \\
\text { optimal }\end{array}$ \\
\hline$[17]$ & Rate-optimal & DL & $\begin{array}{l}\text { Joint power and sub-carrier al- } \\
\text { location }\end{array}$ & Heuristic JSPA algorithm & $\begin{array}{l}\text { The algorithm achieved near- } \\
\text { optimal WSR and user fairness }\end{array}$ \\
\hline
\end{tabular}

objective is to find the optimal power allocation satisfying the constraints. They proposed an algorithm to solve the sub-problem that is based on dynamic programming (DP).

- The multi-carrier power control (MCPC): given a fixed sub-carrier allocation for all sub-carriers, the objective is to maximize the WSR. The problem is formulated as two-stage optimization problem, where in the first stage consists in optimizing the allocated power budget of each sub-carrier, and the second stage performs power allocation on each sub-carrier. To solve the problem, the authors proposed an algorithm based on gradient descent.

An efficient heuristic joint subcarrier and power allocation scheme (called JSPA), was designed by combining the solution of SCUS and MCPC, as presented in figure 3.

The performance of the algorithm was evaluated by numerical simulations and it was compared to with the near-optimal high complexity benchmark scheme lagrangian duality and dynamic programming (LDDP) introduced in [19], and to fractional transmit power control (FTPC) with greedy sub-carrier allocation considered in [19]-[22]. The results showed that JSPA achieves near-optimal WSR and user fairness.

\section{DisCUSSION AND OPEN CHALLENGES}

\section{A. Discussion}

After reviewing all these papers, we saw that each one of them exploited the available resources to achieve a certain goal, and the chosen strategy depends on the situation. In most of them, the proposed solution or algorithm outperformed

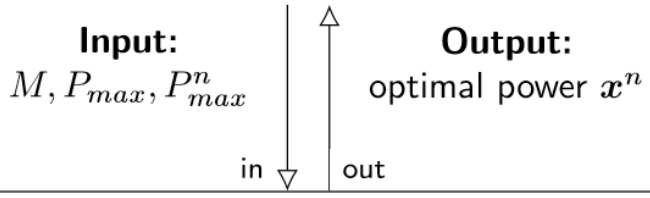

First-stage algorithm: MCPC

Projected gradient descent on each subcarrier's power budget $\bar{P}^{n}$ to maximize WSR $\sum_{n \in \mathcal{N}} F^{n}\left(\bar{P}^{n}\right)$

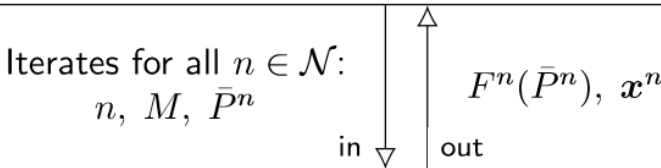

Second-stage algorithm: SCUS

Compute the optimal single-carrier power allocation $\boldsymbol{x}^{n}$ under budget $\bar{P}^{n}$ and constraint $\left|\mathcal{U}_{n}^{\prime}\right| \leq M$

Figure 3. Overview of JSPA [17]

the exiting solutions, but there can be situations, such as in [13], where NOMA was not the preferred candidate, which highlights the importance of the good management of resources depending on the offered service. In order to be able to compare the differences between the papers, we grouped the information in table I, where, for each paper, the type of the link (UL or DL), and the resources that has been treated are specified, the optimization method used to solve the problem is stated along with the main results of the paper. 


\section{B. Open challenges}

In this paper, we only addressed the resource allocation problem for PD-NOMA, but it should be mentioned that $5 \mathrm{G}$ networks are not only based on PD-NOMA.

Several technologies are being studied in order to be integrated with NOMA to fulfill the requirements of $5 \mathrm{G}$ and beyond 5G networks (B5G), such as MIMO and massive MIMO (mMIMO), millimeter wave (mmWave) communications, coordinated multi-point (CoMP) transmission and reception, cooperative communications, etc [2]. The integration of all these different technologies is the topic of research for $5 \mathrm{G}$ and $\mathrm{B} 5 \mathrm{G}$ networks, and lots of these fields are new and not yet well explored, so there exist a huge number of research direction.

In addition, the service-oriented view envisions $5 \mathrm{G}$ systems catering to a wide range of services differing in their requirements and types of devices, and going beyond the traditional human-type communications to include various types of machine-type communications. This requires the network to take different forms depending on the service in question, leading naturally to the notion of slicing the network on a per-service basis. Realizing this service-oriented view requires a radical rethink of the mobile network architecture to turn it into a more flexible and programmable fabric, leveraging technologies like software defined networking (SDN), that relies on the separation between the control plane and the data plane to enhances data forwarding efficiency and network programmability, and NFV, that enables each network function, named virtual network function (VNF), to run on generalpurpose hardware to alleviate deployment costs [23]. Those two last technologies can be used to simultaneously provide a multitude of diverse services over a common underlying physical infrastructure [24].

\section{CONCLUSION}

There is a major difficulty in designing of efficient resource allocation in $5 \mathrm{G}$ networks. In this paper, resource allocation problem using PD-NOMA literature was reviewed, discussed, and classified into two main categories: power/energy-efficient, and rate-optimal. In particular, the paper investigated the objective, optimization methods and the main results of each paper. Furthermore, our paper explores recent solutions on resource allocation problem and provides a comparative table of these solutions. Finally, some open challenges are highlighted, and the mentioned titles could be thoroughly investigated for future research.

\section{ACKNOWLEDGEMENT}

This work is carried out in the framework of the Labex MS2T, which was funded by the French Government, through the program "Investments for the future managed by the National Agency for Research (Reference ANR-11-IDEX0004-02)".

\section{REFERENCES}

[1] Shafi, Mansoor, et al. "5G: A tutorial overview of standards, trials, challenges, deployment, and practice." IEEE journal on selected areas in communications 35.6, 1201-1221, 2017

[2] O. Maraqa, A. S. Rajasekaran, S. Al-Ahmadi, H. Yanikomeroglu, and S. M. Sait, "A Survey of Rate-optimal Power Domain NOMA Schemes for Enabling Technologies of Future Wireless Networks”, Sep. 2019.

[3] M. Aldababsa, M. Toka, S. Gökçeli, G. Karabulut Kurt, and O. Kucur, "A Tutorial on Nonorthogonal Multiple Access for 5G and Beyond", Wireless Communications and Mobile Computing, Jun 2018.

[4] Z. Yuan, G. Yu, and W. Li, "Multi-User Shared Access for 5G," Telecommunications Network Technology, vol. 5, no. 5, pp. 28-30, May 2015.

[5] H. Nikopour and H. Baligh, "Sparse code multiple access," in Proceedings of the IEEE 24th Annual International Symposium on Personal, Indoor, and Mobile Radio Communications (PIMRC '13), pp. 332-336, IEEE, London, UK, September 2013.

[6] R. Hoshyar, F. P. Wathan, and R. Tafazolli, "Novel low-density signature for synchronous CDMA systems over AWGN channel," IEEE Transactions on Signal Processing, vol. 56, no. 4, pp. 1616-1626, 2008.

[7] J. Zhu, J. Wang, Y. Huang, S. He, X. You, L. Yang, ”On Optimal Power Allocation for Downlink Non-Orthogonal Multiple Access Systems", IEEE Areas Commun. 35, 2744-2757, 2017

[8] Z. Wei, D. Wing Kwan Ng, and J. Yuan, "Power-Efficient Resource Allocation for MC-NOMA with Statistical Channel State Information", IEEE Global Communications Conference (GLOBECOM), Dec.2016.

[9] A. MD Shipon, T. Hina, and E. Hossain, "Dynamic user clustering and power allocation for uplink and downlink non-orthogonal multiple access (NOMA) systems", IEEE access, special section on optimization for emerging wireless networks: IoT, $5 \mathrm{G}$ and smart grid communications, Aug. 2016

[10] V. Basnayake, D. N. K. Jayakody, V. Sharma, N. Sharma, P. Muthuchidambaranathan, and H. Mabed, "A New Green Prospective of Nonorthogonal Multiple Access (NOMA) for 5G', Information 2020, 11(2), 89, 2020.

[11] R. Dawadi, S. Parsaeefard, M. Derakhshani, and T. Le-Ngoc, "Powerefficient resource allocation in NOMA virtualized wireless networks", IEEE global communications conference (GLOBECOM), Washington, DC, USA, 2016.

[12] Y. Zhang, "Energy-efficient user scheduling and power allocation for NOMA wireless networks", Oct.2017.

[13] Q. Wu, W. Chen, D. Wing Kwan Ng, and R. Schober, "Spectral and energy efficient wireless powered IoT networks: NOMA or TDMA", IEEE Transactions on Vehicular Technology, vol, 67, issue 7, 2018.

[14] S. Zeb, Q. Abbas, S. A. Hassan, A. Mahmood, R. Mumtaz ; S. M. Hassan Zaidi, S. A. R. Zaidi, and M. Gidlund, "NOMA Enhanced Backscatter Communication for Green IoT Networks", 16th International Symposium on Wireless Communication Systems (ISWCS), 2019.

[15] B. Di, L. Song, and Y. Li, "Sub-channel assignment, power allocation, and user scheduling for non-orthogonal multiple access networks", IEEE transactions on wireless communications, vol. 15, no.11, 2016.

[16] Y. Sun, D. Wing Kwan Ng, Z. Ding, and R. Schober, "Optimal joint power and subcarrier allocation for MC-NOMA systems", Global Telecommunications (GLOBECOM), IEEE Conference and Exhibition, 2016.

[17] L. Salaun, M. Coupechoux, and C. Shue Chen, "Weighted sum-rate maximization in multi-carrier NOMA with cellular power constraint", IEEE INFOCOM, pp.451-459, Apr. 2019

[18] S. Al-Ahmadi, O. Maraqa, M. Uysal, and S. M. Sait, "Multi-user visible light communications: State-of-the-art and future directions,'IEEE Access, vol. 6, pp. 70 555-70 571, Nov. 2018.

[19] L. Lei, D. Yuan, C. K. Ho, and S. Sun, "Power and channel allocation for non-orthogonal multiple access in 5G systems: Tractability and computation," IEEE Trans. Wireless Commun., vol. 15, no. 12, pp. 8580-8594, 2016.

[20] Z. Ding, P. Fan, and H. V. Poor, "Impact of user pairing on 5G non orthogonal multiple-access downlink transmissions," IEEE Trans. Veh. Technol., vol. 65, no. 8, pp. 6010-6023, 2016.

[21] Y. Fu, L. Salaun, C. W. Sung, C. S. Chen, and M. Coupechoux, "Double .. iterative waterfilling for sum rate maximization in multicarrier NOMA systems," in IEEE Int. Conf. Commun. (ICC), 2017.

[22] P. Parida and S. S. Das, "Power allocation in OFDM based NOMA systems: A DC programming approach," in Globecom Workshops, 2014 
[23] R. Su, D. Zhang, R. Venkatesan, Z. Gong, C. Li, F. Ding, F. Jiang, and Z. Zhu. "Resource allocation for network slicing in $5 \mathrm{G}$ telecommunication networks: A survey of principles and models." IEEE Network 33, no. 6, 2019.

[24] X. Foukas, G. Patounas, A. Elmokashfi, and M. K. Marina, "Network Slicing in 5G: Survey and Challenges", IEEE communications magazine, vol.55, iss. 5, 2017. 